\section{Cahiers de Narratologie}

Analyse et théorie narratives

$10.1 \mid 2001$

La voix narrative

\title{
La polyphonie narrative : techniques, fonctions, incidences sur la lecture dans El Árbol de la cienca et La Dama errante de Pìo Baroja
}

\section{Elisabeth Delrue}

\section{(2) OpenEdition \\ Journals}

Electronic version

URL: http://journals.openedition.org/narratologie/6992

DOI: $10.4000 /$ narratologie.6992

ISSN: 1765-307X

Publisher

LIRCES

\section{Printed version}

Date of publication: 1 January 2001

Number of pages: $357-364$

ISBN: 2914561032

ISSN: 0993-8516

Electronic reference

Elisabeth Delrue, "La polyphonie narrative : techniques, fonctions, incidences sur la lecture dans El Árbol de la cienca et La Dama errante de Pì Baroja", Cahiers de Narratologie [Online], 10.1 | 2001, Online since 20 November 2014, connection on 23 February 2021. URL: http://journals.openedition.org/ narratologie/6992 ; DOI: https://doi.org/10.4000/narratologie.6992 


\title{
LA POLYPHONIE NARRATIVE : TECHNIQUES, FONCTIONS, INCIDENCES SUR LA LECTURE DANS EL ARBOL DE LA CIENCIA ET LA DAMA ERRANTE DE PÍO BAROJA
}

\author{
Elisabeth DELRUE \\ Université d'Amiens
}

La narrativité dans les romans de Pío Baroja foisonne, on le sait, de personnages et d'événements. Analyser la voix narrative, à savoir qui raconte ? dans deux de ces romans, $E l$ Árbol de la ciencia, publié en 1911 et La dama errante de 1908 qui avec La ciudad de la niebla de 1909 constituent la trilogie de $L a$ Raza conduit irrémédiablement à parler de phénomènes de polyphonie narrative. En effet, on y observe sans mal la fragmentation de l'instance énonciative en plusieurs voix narratives servant de relais énonciatif au narrateur principal, en se plaçant à l'origine d'un récit enchâssé, où s'insèrent parfois des répliques en style direct, l'émergence de récits ironiques, l'interpolation de fragments de discours hétérogènes (textes de lettres reproduites dans leur intégralité, articles de journaux fidèlement retranscrits).

Notre communication va donc examiner globalement tous ces phénomènes mis en œuvre dans les deux romans, analyser les fonctions que l'auteur leur assigne, cerner, enfin, les incidences sur la lecture qu'ont de tels procédés.

A première vue, le récit de La Dama errante est pris en charge par un narrateur extérieur de troisième personne, le fameux narrateur omniscient qui commente et juge, parfois par le biais de réflexions ironiques et tendancieuses, introduit des répliques en style direct ou indirect, fait appel au discours indirect libre et au discours narrativisé ou raconté et délègue sa voix à des personnages intradiégétiques qui, à leur tour, 
rapportent un nouveau récit : el doctor Aracil1 (p. 75 en style direct ; p. 95, p. 188 et suivantes), Maria (p. 80 et suivantes), el Grillo (p. 168-169), don Álvaro (p. 174, 178-179). Mais, ce n'est pas tout. Des textes de nature spécifique sont interpolés : des articles de journaux, (p. 97, 98, 119-122, 129-130), la lettre reproduite dans son intégralité de l'anarchiste Nilo Brull (p. 125-129).

Dans El Árbol de la ciencia, le récit est majoritairement à focalisation zéro, mais le narrateur n'est pas neutre et délègue parfois, lui aussi sa voix à des personnages intradiégétiques. Plusieurs cas se dessinent : Lulu retrace ses péripéties (pages $78,210-211)^{2}$, Iturrioz cite un passage de la Bible (pages 131132). Plus loin, il rapporte une scène à laquelle il a assisté à Cuba (page 199), Andrés narre sa visite dans une maison de prostitution (page 219). Venancia raconte une histoire (pages 85-87) où elle joue un rôle d'héroïne et d'observatrice.

Pour cerner les fonctions de ces phénomènes de polyphonie narrative tenons-nous - en à ce que déduit Maurice Couturier, dans sa quête de la figure auctoriale :

«Pour le romancier moderne, l'enjeu principal consiste donc à imposer son autorité figurale à un texte dont il feint de se désolidariser (...). Le roman moderne sera donc habité par plusieurs énonciateurs entre lesquels l'auteur réel distribuera ses effets de voix et aussi ses désirs, rendant ainsi le lecteur incapable de reconstituer à coup sûr les contours du « sujet-origine » pour reprendre l'expression de Käte Hamburguer. ${ }^{3}$

Les sources d'énonciation hétérogènes qui s'enchevêtrent dans les deux romans répondraient, à nos yeux et d'après ce qui précède, à une stratégie de fuite utilisée par Baroja pour déconnecter le discours narratif par rapport à son discours personnel et donner l'impression que le roman est écrit par

${ }^{1}$ Les numéros de pages cités correspondent à l'édition: BAROJA Pío, La Dama errante, Madrid, Editorial Caro Raggio, 1974, 234 p.

${ }^{2}$ Les numéros de pages cités correspondent à l'édition suivante : BAROJA Pío El Árbol de la ciencia, Madrid, Alianza editorial, 1986, 250 p., El libro de Bolsillo.

${ }^{3}$ Maurice COUTURIER, La figure de l'auteur, Paris, Seuil, 1995, p. 73 . 
plusieurs locuteurs, qui se distinguent de sa figure, aux trois niveaux où habituellement s'inscrivent la subjectivité, le plan sémantique, le plan syntaxique et le plan pragmatique, afin de piéger le lecteur réel que nous sommes, à savoir le lecteur en chair et en os qui peut ou non remplir le rôle assigné par les sollicitations textuelles.

Jaap Lintvelt dans Essai de typologie narrative ${ }^{4}$, établit des distinctions entre auteur abstrait - auteur concret, et narrateur-auteur concret. Pour lui, l'auteur concret, personnalité historique et biographique en chair en os, en dehors du monde littéraire, est le créateur réel de l'œuvre littéraire dont le nom figure sur le livre. L'auteur abstrait ou « scripteur» ou " auteur implicite » pour Booth ou « auteur modèle » pour Umberto Eco est la projection littéraire de l'auteur concret. Inclus dans l'œuvre littéraire, sans y être représenté directement, il produit un monde romanesque qu'il transmet à son destinataire. Cet auteur abstrait est à l'origine de la création du narrateur, qui, à son tour, est à l'origine de la création du récit.

Dans les deux romans, une instance narrative anonyme " auteur-narrateur » distincte de l'auteur comme " personne biographique » prend en charge le récit principal et cherche, par plusieurs procédés, à brouiller ou à franchement gommer les traces de sa présence.

Dès le péritexte, à savoir ici, les titres des parties et des chapitres, la voix qui assume leur énonciation s'efforce de viser la neutralité absolue. Majoritairement thématiques, selon la terminologie de Genette, ils ont un caractère elliptique et un style nominal qui ne laissent entrevoir aucun investissement subjectif du locuteur. Quand parfois un verbe intervient, il est au passé simple, à l'imparfait ou au présent aoristique (ou historique), c'est-à-dire aux temps qui relèvent de l'énonciation historique selon Benvéniste, où toute trace patente de l'activité du locuteur est proscrite.

Dans le corps même du récit, cette tendance se confirme à plusieurs niveaux. D'abord, le mode d'énonciation narrative retenu dans les deux romans, se donne d'emblée comme

4 Jaap LINTVELT, Essai de typologie narrative, Paris, Corti, 1981, pp. 24-28. 
dissocié de la situation d'énonciation et les événements se présentent comme se racontant eux-mêmes. En effet, l'histoire est racontée à la troisième personne, au passé simple et à l'imparfait avec des passages au présent de l'indicatif. Or, la troisième personne d'après Benvéniste est la non - personne, c'est-à-dire, celle qui n'implique aucune personne. Elle peut prendre n'importe quel sujet ou n'en comporter aucun et le passé simple est "le temps de l'événement hors de la personne du narrateur ». L'imparfait, on le sait, relève à la fois de l'énonciation historique 5 et de l'énonciation discursive. Quand le narrateur porte des jugements malveillants, comme nous l'avons signalé, il ne parle pas au nom de l'auteur concret. Sa critique est vouée à la prudence par le biais de l'anonymat de la troisième personne ou du collectif auquel il s'intègre et dont il partage l'orientation critique. Cette troisième personne se fond, en effet, dans une pluralité indécise et se donne comme le porte-parole d'une communauté à laquelle est censé appartenir le destinataire idéal du livre. C'est le cas, dès l'incipit de La Dama errante, où se multiplient les occurrences de l'adjectif possessif "nuestro": «nuestra época», «nuestro país», «nuestros tiempos». Le déictique temporel "hoy ", employé dans la même page qui d'ordinaire introduit une perspective temporelle depuis laquelle se relate le récit, ici, à nos yeux, se borne à circonscrire un champ déictique partagé par le lecteur et renvoie à «nuestra época, nuestro tiempo ».

L'émergence d'une première personne du singulier au travers d'un je, déjà fort rare en soi est là pour assumer la totalité d'un énoncé non critique, comme la provenance incertaine d'une autorité ou d'une référence citées, par exemple. Dans La Dama errante, on en a, par deux fois l'illustration :

- «Un escritor, creo que Stendhal (...)»6

5 Émile BENVENISTE, Problèmes de linguistique générale, I, Paris, Gallimard, 1966, p. 239.

${ }^{6}$ Pio BAROJA, La Dama errante, Madrid, Editorial Caro Raggio, 1974 p. 32. 
La polyphonie narrative : techniques, fonctions, incidences

sur la lecture dans El Arbol de la ciencia et La Dama errante

- « hay en un libro viejo, cuyo nombre no recuerdo (...) $\gg^{7}$

La prudence de la critique est accentuée par le rôle particulier assigné à l'imparfait. En effet, au lieu d'énoncer ses condamnations au présent, c'est-à-dire au temps qui correspondrait à son acte énonciatif, le narrateur les émet à l'imparfait, c'est-à-dire qu'il les fond dans l'ensemble des descriptions du monde extérieur, de l'évocation des faits habituels, des pensées des personnages et des procès extérieurs à la dynamique narrative.

En outre, le présent ici utilisé ne correspond nullement à l'acte énonciatif du narrateur mais confère à son énoncé une portée générale. Les vérités atemporelles généralisantes ou maximes qui prennent appui sur une idée, une action, le caractère d'un personnage pour énoncer les lois sous lesquelles viennent se ranger les faits particuliers sont des commentaires idéologiques, neutres, objectifs, qui effacent les marques de l'énonciateur pour leur substituer la voix inattribuable de la doxa ou discours de véridiction.

Par le biais de l'ironie, enfin, le narrateur fait entendre dans sa parole le point de vue d'un «énonciateur » dont il se distancie. Le recours à cette combinaison paradoxale dans la même énonciation manifeste en soi une volonté de brouiller la source énonciative.

Quant aux modalités de gestion de la parole de l'Autre (style direct, style indirect, style indirect libre, discours narrativisé ou raconté), là encore l'instance narrative anonyme recherche, selon les cas, une hétérogénéité ou une ambiguïté énonciative.

Le style direct est une forme pour l'auteur de ne pas assumer l'énonciation de son texte parce qu'il établit une démarcation nette entre les propos rapportés, rattachés à une autre situation d'énonciation définie par les coordonnées spatio-temporelles d'un locuteur distinct et la locution narrative.

${ }^{7}$ Ibidem, p. 69. 
Par ailleurs, dans son analyse des modalités d'insertion de la parole de l'Autre, C. Kerbrat-Orecchioni ${ }^{8}$ distingue deux classes parmi les informations véhiculées par les verbes de locution, celles qui ont une valeur descriptive, celles qui impliquent un jugement de valeur de l'énonciateur. Or, dans nos deux romans, le style direct est le type de discours rapporté dominant et, souvent, c'est le verbe descriptif « dijo » qui l'introduit quand ce n'est pas le contexte qui seul suffit à marquer le changement d'espace énonciatif. Nombreuses, en effet, sont les séquences dialoguées où, souvent les répliques brèves et interdépendantes ne sont guère escortées d'un verbe de parole qui les précède, les interrompt ou les suit.

Le style indirect est aussi à l'œuvre dans les deux livres. Or, le principe de la double voix s'y opère et génère une ambiguïté énonciative. Effectivement, l'énonciateur second qui rapporte les paroles de l'énonciateur premier après leur avoir fait subir une suite de transformations cherche à interpréter les effets de voix de son interlocuteur et à en dégager le sens plutôt qu'à les rapporter fidèlement. Il s'interpose en tant que réflecteur, tout en semblant donner la parole à ses créatures. Si bien qu'en l'absence de marques de distanciation explicite, le destinataire ne sait s'il doit attribuer au rapporteur ou au locuteur originel les traces de subjectivité et les mots employés.

Le style indirect libre auquel fait appel l'auteur échappe, lui aussi, à une identification univoque. Il s'agit d'une énonciation dédoublée, où le rapporteur simule une prise de parole dont en réalité, un autre que lui-même est responsable, de sorte que le lecteur n'est pas assuré de l'endroit exact où ce discours surgit et du moment précis où il disparaît.

Enfin, l'utilisation particulière du discours narrativisé ou raconté participe aussi de la quête d'une ambiguïté énonciative. La parole du locuteur y est fondue au discours du narrateur qui la fait sienne et, bien souvent, dans ce cas de figure, une phrase sans traces de modalisation pose la question de la source énonciative. C'est le cas, par exemple

${ }^{8}$ Catherine KERBRAT-ORECCHIONI, L'énonciation de la subjectivité dans le langage, Paris, A. Colin, 1980, p. 115. 
La polyphonie narrative : techniques, fonctions, incidences

sur la lecture dans El Arbol de la ciencia et La Dama errante

dans El Árbol de la ciencia quand Venancia raconte une histoire, au chapitre VII de la seconde partie. Le récit est d'abord pris en charge par le narrateur omniscient, il glisse ensuite au discours direct et finit, sans doute, sur un discours narrativisé dont on a du mal à identifier l'énonciateur.

Si par cette palette de procédés, l'auteur implicite donne l'illusion de laisser vivre le monde romanesque qu'il décrit de l'extérieur, en simulant l'objectivité ou en fondant son discours critique à celui de la collectivité dont ferait partie le destinataire idéal des romans, ou en rendant difficile son attribution par brouillage de sources énonciatives univoques, dans les fragments de textes interpolés, parfois dépourvus de statut littéraire, il vise, en outre, à faire croire qu'ils ont été écrits par d'autres que lui.

De fait, en cherchant à faire assumer à ses personnages la tâche de raconter leur propre histoire ou des faits dont ils ont été témoins, souvent en style direct, l'auteur, là encore s'emploie à faire oublier son implication dans le processus énonciatif : il tend à maintenir l'illusion d'une identité entre scripteur, narrateur et personnage, en particularisant le style des locuteurs (le registre lexical, les figures de rhétorique, les formules affectives et modalisatrices) pour renforcer l'illusion d'une singularité et d'une différence. L'impression est ainsi donnée que le roman est écrit par plusieurs personnes, qui de plus se distinguent de la figure de l'auteur, pourtant à l'origine du maniement de tous ces phénomènes textuels. Il en est de même pour les extraits de journaux ou la lettre de l'anarchiste Nilo Brull dans La Dama errante, en particulier. Les articles reproduits, de fait, se caractérisent par un langage essentiellement référentiel aux allures de compte rendu, dépourvu de jugements subjectifs et d'expressions évaluatives, mais riche en formes impersonnelles et en modalisateurs. La lettre de Brull, intégralement retranscrite affiche, au contraire, l'emphase spécifique des discours anarchistes de l'époque avec ses hyperboles multiples, ses antithèses, ses exclamations et ses images.

Si ces deux romans mosaïques où une voix en donne à entendre plusieurs autres, par le simulacre d'un collage de fragments discursifs à style propre ont donc été écrits, pour gommer l'empreinte de l'énonciateur premier et masquer son 
rôle essentiel dans l'orchestration des phénomènes textuels, ils ont été surtout conçus, et c'est ce que nous allons voir à présent, en vue de piéger le lecteur, nous semble-t-il à trois niveaux.

Dans les œuvres examinées, on entend certains personnages émettre des critiques de l'Espagne et de ses habitants qui font écho aux jugements portés par le narrateur omniscient. Partiellement redondantes les unes avec les autres, ces évaluations courent d'un bout à l'autre des textes et constituent ainsi l'axiologie des valeurs manipulées par le récit qui finit par sourdre de la confrontation des diverses voix, sans qu'elle soit directement imputable à l'auteur concret Baroja. L'objectif de ce dernier, dans le maniement de la polyphonie est donc d'asséner avec plus de force une idéologie subversive sans qu'elle lui soit directement attribuée dans la mesure où il parle par la voix d'un autre ou qu'il s'ingénie à rendre sa figure d'auteur évanescente.

En outre, la participation du lecteur est activement sollicitée par cette vision kaléidoscopique de la réalité dont il doit reconstituer la cohérence par des recoupements multiples ou relativiser les perspectives parfois contradictoires.

Enfin, la polyphonie à l'œuvre dans les deux romans vise, à notre avis, l'illusion référentielle qui contribue à piéger le lecteur, en lui donnant l'impression que le personnage est vivant et autonome puisqu'il est à même de prendre en charge sa propre histoire et celle des autres. 